issues: a code structure that allows low encoding complexity, and a flexible ratecompatible code that allows matching to various channel conditions. Most of the previous high-performance LDPC codes for the relay channel are tightly optimized for a given channel quality, and are not easily adapted without extensive re-optimization for various channel conditions. This code for the relay channel combines structured design and easy encoding with rate compatibility to allow adaptation to the three links involved in the relay channel, and furthermore offers very good performance. The proposed code is constructed by synthesizing a bilayer structure with a protograph. In addition to the contribution to relay encoding, an improved family of protograph codes was produced for the pointto-point AWGN (additive white Gaussian noise) channel whose high-rate mem- bers enjoy thresholds that are within 0.07 $\mathrm{dB}$ of capacity.

These LDPC relay codes address three important issues in an integrative manner: low encoding complexity, modular structure allowing for easy design, and rate compatibility so that the code can be easily matched to a variety of channel conditions without extensive re-optimization. The main problem of half-duplex relay coding can be reduced to the simultaneous design of two codes at two rates and two SNRs (signal-to-noise ratios), such that one is a subset of the other. This problem can be addressed by forceful optimization, but a clever method of addressing this problem is via the bilayer lengthened (BL) LDPC structure. This method uses a bilayer Tanner graph to make the two codes while using a concept of "parity forwarding" with subsequent successive decod- ing that removes the need to directly address the issue of uneven SNRs among the symbols of a given codeword. This method is attractive in that it addresses some of the main issues in the design of relay codes, but it does not by itself give rise to highly structured codes with simple encoding, nor does it give rate-compatible codes. The main contribution of this work is to construct a class of codes that simultaneously possess a bilayer parity-forwarding mechanism, while also benefiting from the properties of protograph codes having an easy encoding, a modular design, and being a rate-compatible code.

This work was done by Dariush Divsalar of Caltech, and Thuy Van Nguyen and Aria Nosratinia of the University of Texas at Dallas for NASA's Jet Propulsion Laboratory. For more information, contact iaoffice@jpl.nasa.gov. NPO-47539

\title{
Influence of Computational Drop Representation in LES of a Droplet-Laden Mixing Layer
}

\section{For numerical simulations of such flows, fine-grid LES is not as accurate as coarse-grid LES.}

\section{NASA's Jet Propulsion Laboratory, Pasadena, California}

Multiphase turbulent flows are encountered in many practical applications including turbine engines or natural phenomena involving particle dispersion. Numerical computations of multiphase turbulent flows are important because they provide a cheaper alternative to performing experiments during an engine design process or because they can provide predictions of pollutant dispersion, etc. Two-phase flows contain millions and sometimes billions of particles. For flows with volumetrically dilute particle loading, the most accurate method of numerically simulating the flow is based on direct numerical simulation (DNS) of the governing equations in which all scales of the flow including the small scales that are responsible for the overwhelming amount of dissipation are resolved. DNS, however, requires high computational cost and cannot be used in engineering design applications where iterations among several design conditions are necessary. Because of high computational cost, numerical simulations of such flows cannot track all these drops.

The objective of this work is to quantify the influence of the number of computational drops and grid spacing on the accuracy of predicted flow statistics, and to possibly identify the minimum number, or, if not possible, the optimal number of computational drops that provide minimal error in flow prediction. For this purpose, several Large Eddy Simulation (LES) of a mixing layer with evaporating drops have been performed by using coarse, medium, and fine grid spacings and computational drops, rather than physical drops. To define computational drops, an integer $N_{\mathrm{R}}$ is introduced that represents the ratio of the number of existing physical drops to the desired number of computational drops; for example, if $N_{\mathrm{R}}=8$, this means that a computational drop represents 8 physical drops in the flow field. The desired number of computational drops is determined by the available computational resources; the larger $N_{\mathrm{R}}$ is, the less computationally intensive is the simulation. A set of first order and second order flow statistics, and of drop statistics are extracted from LES predictions and are compared to results obtained by filtering a DNS database. First order statistics such as Favre averaged stream-wise velocity, Favre averaged vapor mass fraction, and the drop stream-wise velocity, are predicted accurately independent of the number of computational drops and grid spacing. Second order flow statistics depend both on the number of computational drops and on grid spacing. The scalar variance and turbulent vapor flux are predicted accurately by the fine mesh LES only when $N_{\mathrm{R}}$ is less than 32 , and by the coarse mesh LES reasonably accurately for all $N_{\mathrm{R}}$ values. This is attributed to the fact that when the grid spacing is coarsened, the number of drops in a computational cell must not be significantly lower than that in the DNS.

Results indicate that for large $N_{\mathrm{R}}$ values, the fine-grid LES is not as accurate as coarse-grid LES, besides being computationally more intensive. This is attributed to the fact that a fine-grid LES used in conjunction with a reduction in the number of followed drops from the physical to a computational drop field implies that there is necessarily a smaller number of drops in a computational cell than in DNS; this aspect naturally influences the flow development and biases it from the filtered DNS. The key to success seems to be having approximately the same and not a significantly smaller number of drops in the computational cell volume in LES as compared to those 
in DNS. Thus, the notion that smooth statistics are all that is required in the choice of the number of computational drops does not hold. This is because unlike particles in a Monte-Carlo calculation where the number of these tracked particles would be increased to obtain convergence of results, drops are not stochastic particles as they obey a deterministic trajectory that is only modulated by turbulence. This finding also highlights the importance of obtaining drop-number experimental data in combustion chambers or natural flows.
This work was done by Josette Bellan and Senthilkumaran Radhakrishnan of Caltech for NASA's Jet Propulsion Laboratory. For more information, contact iaoffice@jpl.nasa.gov. NPO-48202 